\section{G324(P) ABSTRACT WITHDRAWN}

\section{G325(P) TO ERR IS HUMAN: THEMATIC CHARACTERISATION OF PAEDIATRIC CLINICAL INCIDENTS WITHIN A LARGE DISTRICT GENERAL HOSPITAL}

S Li, H Sadreddini, J Surridge. Derby Children's Hospital, Royal Derby Hospital, Derby, UK

10.1136/archdischild-2015-308599.301

Aims Our children's hospital encompasses several acute clinical areas. These include paediatric medical and surgical inpatient wards (including the children's high dependency ward), the children's emergency department and the neonatal intensive care unit. Collectively these are busy and challenging environments where the provision of care is increasingly complex. These provide opportunities for errors to occur making unintended consequences to harm more likely. The Francis report published in February 2013 highlighted the need for openness and transparency in regards to patient safety. We reviewed clinical incidents reported within all acute clinical paediatric areas in order to characterise commonly recurring themes.

Method Clinical incident forms from all acute paediatric clinical areas from 1 September 2012 until 31 August 2014 were retrospectively reviewed. Incidents were assessed for the degree of actual harm caused to patients. They were subsequently categorised according to the National Reporting and Learning System incident types for each clinical area. Medications incidents were further sub-classified by type.

Results 872 incident forms involving all acute paediatric clinical areas were submitted over a 24 month period. $67 \%$ of all clinical incidents were reported as having insignificant harm to patients and only $2 \%$ as catastrophic. The types of incidents reported are shown in Figure 1. Medication errors accounted for a significant percentage of reported incidents within each clinical area (19\% children's emergency department; 30\% children's wards; $26 \%$ neonatal intensive care unit). Other commonly reported incidents were infrastructure problems and patient

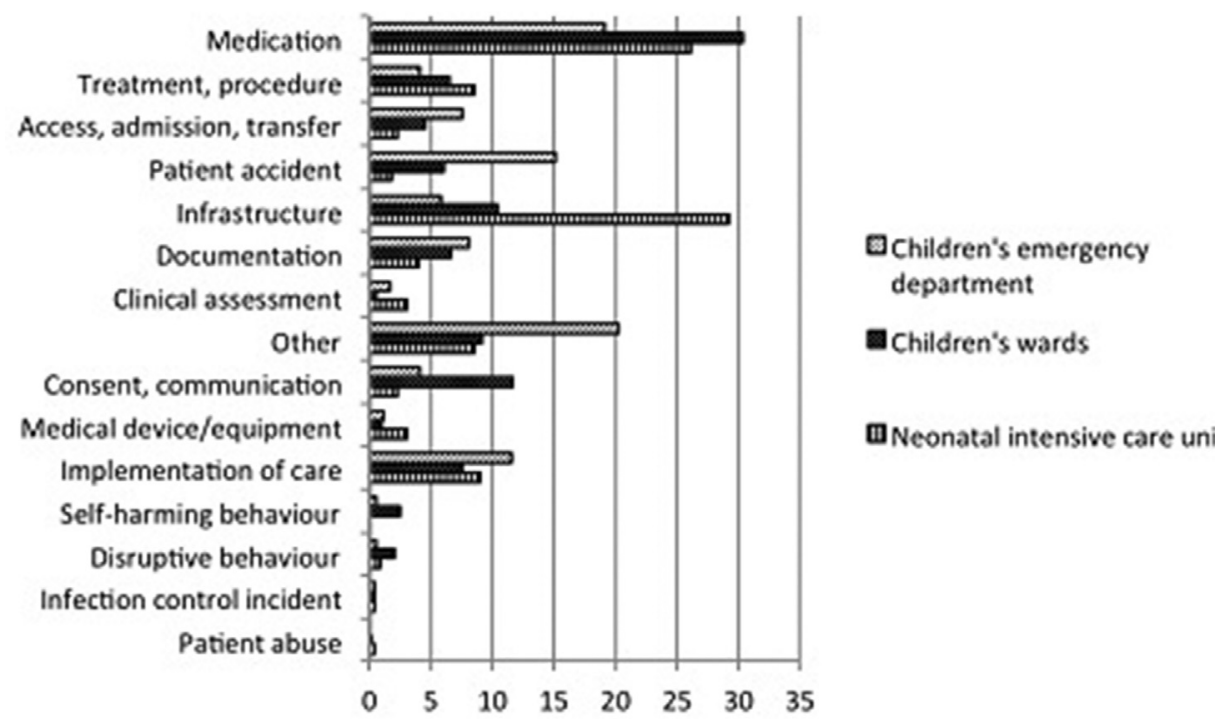

Abstract G325(P) Figure 1 Percentage of reported incident types

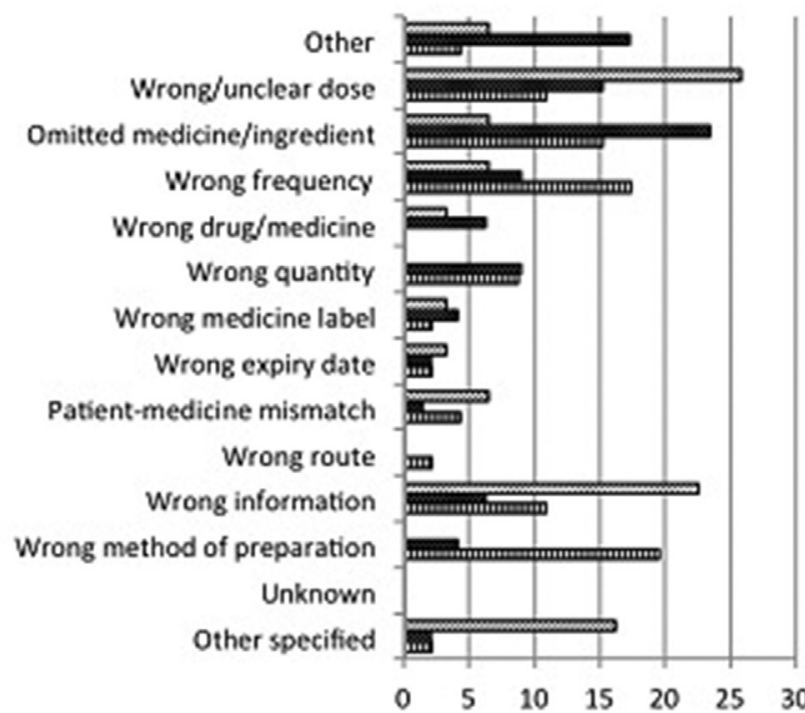

Whildren's emergency
department

Children's wards

四eonatal intensive care uni

Abstract G325(P) Figure 2 Percentage of medication incidents by specified types 
accidents. The types of medication incidents reported are shown in Figure 2. The most commonly reported medication incidents within each clinical area were wrong dose of medicine $(26 \%$ children's emergency department), omitted medicines (23\% children's wards) and wrong method of preparation (20\% neonatal intensive care unit).

Conclusion Incident forms submitted within a children's hospital should be reviewed so that commonly recurring themes can be identified. Within our hospital medication errors accounted for the majority of reported incidents. Characterisation of these themes have implications for the training of staff which can be tailored to individual paediatric clinical areas.

\section{G326(P) CASESERIES: ALL THAT WHEEZES IS NOT ASTHMA}

${ }^{1} F$ Roked, ${ }^{1} \mathrm{O}$ Narayan, ${ }^{1} \mathrm{P}$ Kenia, ${ }^{2} \mathrm{D}$ Parikh, 'S Rao. 'Respiratory, Birmingham Children's Hospital, Birmingham, UK; 'Paediatric Surgery, Birmingham Children's Hospital, Birmingham, UK

\subsection{6/archdischild-2015-308599.302}

Aims To illustrate that although asthma is a common cause for wheezing, there should be high index of suspicion for rarer causes when either the presentation or the response to treatment is atypical.

Methods Retrospective review of case notes.

Results A 12 year old girl had worsening shortness of breath and wheeze on minimal exertion over a year. She had been diagnosed with asthma and received multiple courses of oral and inhaled steroids with minimal improvement. On chest auscultation she had an inspiratory and expiratory wheeze, more noticeable when she laughed. A chest $\mathrm{x}$-ray showed bulbous widening of the superior mediastinum (Figure 1a).

A flow volume loop suggested extrinsic intrathoracic airway compression. A computed tomography (CT) scan showed a mediastinal cystic mass compressing the distal trachea suggesting a foregut duplication (Figure 1b); this was removed thoracoscopically.

A 3 year old girl diagnosed with asthma by her GP presented with a worsening cough and wheeze for 3 months. She presented with an exacerbation of symptoms. On examination there was a biphasic wheeze on lying flat. Due to foreign travel, a chest $\mathrm{x}$-ray was requested revealing a large foreign body
(Figure 2a). A button battery was retrieved endoscopy from her oesophagus. There was no tracheoeophageal fistula found on rigid bronchoscopy.

A 4 year old girl presented with a worsening wheeze and wet cough for 1 year. She had received antibiotics and inhalers from her GP. On examination, there was decreased air entry unilaterally. A chest X-ray showed an extensive opacity in the left hemithorax causing mediastinal shift (Figure 3a). A CT scan showed a large cystic mass (Figure $3 \mathrm{~b}$ ). This was a cystic teratoma requiring resection. A 1 year old girl had a similar history, findings on examination and imaging to this, which was also a teratoma (Figure 4a).

Conclusion Routine imaging is not required to assess asthma, but if symptoms and signs are unusual or there is worsening of symptoms despite treatment, it should be considered, to seek alternative diagnoses. Assessment of asthma should include questioning the diagnosis at every clinic review as 'all that wheezes is not asthma'.

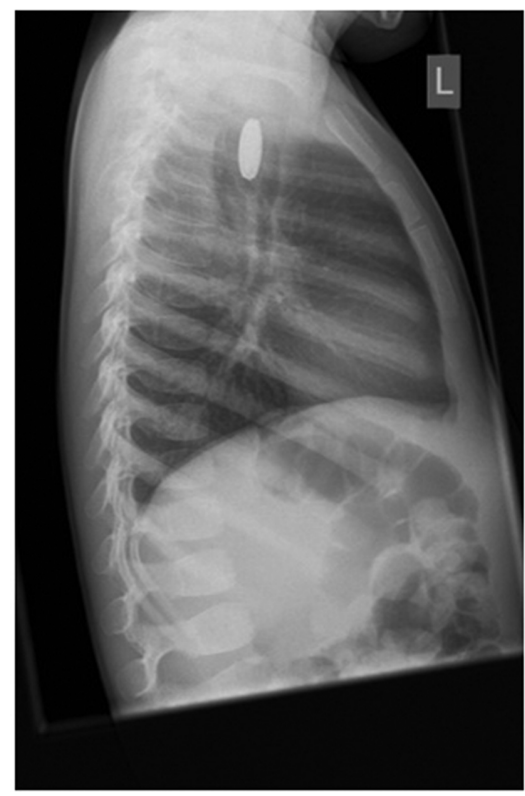

Abstract G326(P) Figure 2a Foreign body in oesophagus
Image 1a: Widened Mediastinum

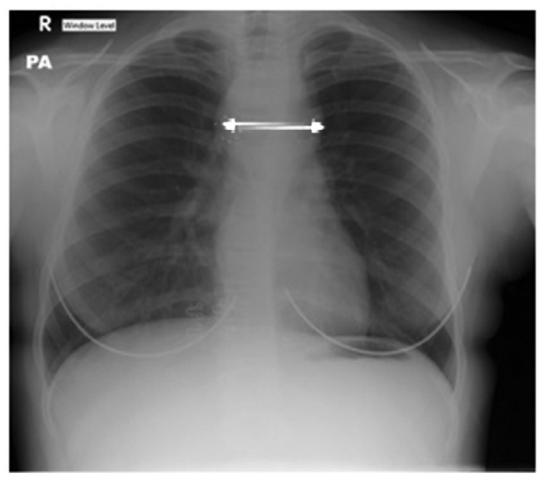

Image 1b: Foregut duplication cyst

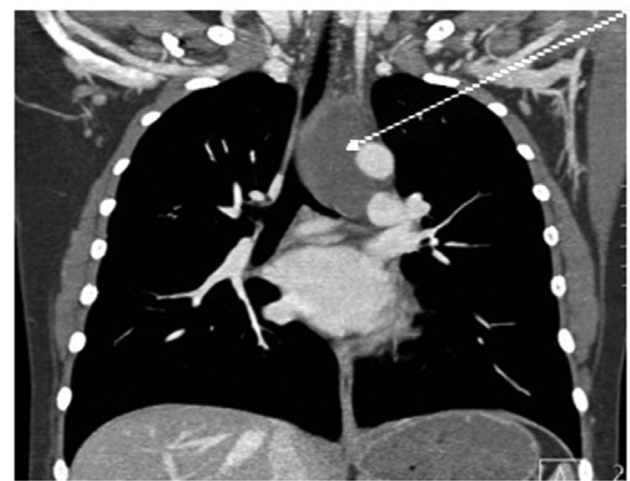

Abstract G326(P) Figure 1b Foregut duplication cyst 